\title{
Longitudinal nasal meticillin-resistant Staphylococcus aureus colonisation among resident physicians at an urban, public hospital
}

\author{
Jennifer Carnell, Gene Hern, Amandeep Singh, \\ Larry Lambert, Paul Cheung, Bitou Cisse, Charlotte Silver, Bradley Frazee \\ Alameda County Medical Center, Highland Hospital, Oakland, California, U.S.A.
}

doi: 10.3396/ijic.V7i3.025.11

\begin{abstract}
Introduction
Understanding the epidemiology of meticillinresistant Staphylococcus aureus (MRSA) nasal colonisation among healthcare providers is essential for development of effective MRSA infection control strategies. There are no studies of nasal colonisation exclusively in resident physicians, a group of mobile healthcare providers who spend many hours in hospitals, have significant direct patient contact, and frequently work in multiple areas of the hospital. Furthermore, studies using annual surveillance to examine longitudinal trends in Staphylococcus aureus (S. aureus) nasal colonisation of healthcare workers are scarce.
\end{abstract}

We examined longitudinal rates of MRSA and meticillin-sensitive Staphylococcus aureus (MSSA) nasal colonisation in resident physicians by performing annual surveillance cultures.

\section{Methods}

We conducted a prospective, observational study of $S$. aureus nasal colonisation among resident physicians at an urban, public teaching hospital in Oakland, California, U.S.A. Samples were collected from residents in the emergency medicine, general surgery, internal medicine and oral maxillofacial surgery residency programs annually between June and January of 2006, 2007, and 2008. All residents were eligible and were enrolled if they provided informed consent. The hospital institutional review board approved the study.

Nasal swabs (BBL Culture Swab Plus collection and transport system) from study participants underwent culture and susceptibility testing, including selective MRSA culture. Swabs were directly inoculated on to each of three media in the following order: Mannitol Salt Agar with $6.5 \% \mathrm{NaCl}$ and $4 \mathrm{mcg} / \mathrm{ml}$ oxacillin

\section{Corresponding author}

Jennifer Carnell

Alameda County Medical Center, Highland Hospital

1411 East $31^{\text {st }}$ Street, Oakland, California 94602, U.S.A.

Email: jencarnell@gmail.com

Work phone: 510-437-4564

Mobile: 510-225-8579

Fax: 510-437-8322 
(MSA-OX), Tryptic Soy Agar with 5\% sheep blood (TSA-BL), and Rose agar (Hardy Diagnostics, Santa Maria, CA). TSA-BL plates were incubated in a $7 \%$ $\mathrm{CO}_{2}, 35^{\circ} \mathrm{C}$ incubator; MSA-OX and Rose plates were incubated in a $35^{\circ} \mathrm{C}$ ambient air incubator; all plates were incubated for 18-24 hours prior to initial evaluation and held up to 48 hours before being reported as negative for MRSA.

Identification of $S$. aureus, including MRSA, was confirmed by coagulase testing (tube and slide) and multi-substrate biochemical panels using the MicroScan WalkAway testing platform and positive combo microtiter trays. All MicroScan vancomycin data were confirmed by AB Biodisc E-tests.

\section{Data Analysis}

The proportion of residents testing positive for nasal S. aureus colonisation and $95 \%$ confidence intervals (Cls), using exact confidence intervals, were calculated.

\section{Results}

During the three-year study period, a total of 441 samples were obtained from 262 resident physicians. Depending on the study year, the total number of resident physicians in the emergency medicine, internal medicine, general surgery and oral maxillofacial surgery residency programs combined varied between 171 and 179. Each study year, 147 residents participated meaning that, at a minimum, $82 \%$ of the eligible population participated each year. Overall, $S$. aureus was detected in 13 subjects $(5.0 \%, 95 \% \mathrm{Cl} 2.7$ $8.3 \%)$, MRSA in $8(3.1 \%, 95 \% \mathrm{Cl} 1.3-5.9 \%)$, and MSSA in $5(1.9 \%, 95 \% \mathrm{Cl} 0.6-4.4 \%)$. The annual prevalence of $S$. aureus/MRSA nasal colonisation in our resident physicians was $4.8 \% / 2.0 \%$ in $2006,3.4 \% / 2.7 \%$ in 2007 , and $2.0 \% / 2.0 \%$ in 2008 .

Longitudinal culture results were available for the 120 subjects who were tested annually for two or three consecutive years. Overall, there were four subjects who were initially culture-negative but acquired colonisation (3 MRSA, 1 MSSA) during the study whereas there were five instances of apparent loss of colonisation (3 MRSA, 2 MSSA).
As the total number of years in training increased (from 1 to 5), the proportion of resident physicians colonised with MRSA increased from $1.2 \%$ (95\% $\mathrm{Cl} 0.2-4.4 \%$ ) to $8.7 \%(95 \% \mathrm{Cl} 1.1-28.0 \%)$.

\section{Discussion}

The annual prevalence of $S$. aureus nasal colonisation in our resident physicians was only 2.0-4.8\% compared with recent large studies that found a $S$. aureus prevalence of $27.1 \%$ in the U.S. population ${ }^{1}$ and an MSSA prevalence of $23.7 \%$ in healthcare providers. $^{2}$ The extremely low prevalence of MSSA nasal colonisation in our subjects accounts for the difference in overall $S$. aureus colonisation between our study and others; this is surprising given that MSSA is a normal nasal coloniser. While it has been shown that healthcare workers are less likely to be colonised with $S$. aureus than community members due to a lower prevalence of MSSA colonisation, the overall $S$. aureus rate in our sample is still an order of magnitude lower than expected. ${ }^{3}$

Of the subjects colonised with $S$. aureus, two thirds were colonised with MRSA and one third with MSSA. To our knowledge, no other study has found MRSA colonisation to exceed MSSA colonisation. In one study, nasal $S$. aureus isolates from healthcare workers were almost twice as likely to be MRSA as those from community members, however MSSA was still roughly three times as prevalent as MRSA. ${ }^{3}$ In a longitudinal study of $S$. aureus nasal colonisation in the U.S. from 2001 to 2004, MSSA nasal colonisation decreased by $3.8 \%$ while colonisation with MRSA nearly doubled. ${ }^{1}$ Our findings may reflect a continuation and magnification of this trend among healthcare providers.

MRSA colonisation steadily increased as residents progressed through training, suggesting, as other studies have, that increasing duration of time in patient care is a risk factor for colonization. ${ }^{4,5}$

Our longitudinal data suggest that MRSA colonisation, like MSSA colonisation, is dynamic. Nine of the thirteen residents who were $S$. aureus positive either 
acquired or lost colonisation during the three-year study period.

\section{Limitations}

Our results, obtained at a single, urban, public teaching hospital serving a population with a high rate of MRSA SSTIs and colonisation, may not be applicable to other populations or hospitals. ${ }^{6}$ We did not collect information regarding whether study participants underwent treatment for MRSA positive nasal swabs during the study.

\section{Conclusions}

Our findings suggest that residents have the potential to play a role in MRSA transmission. The absolute duration of MRSA colonisation is unknown as residents were sampled only once annually. Future studies incorporating more frequent cultures might clarify whether a larger proportion of the resident physician population is transiently colonised and better define the usual duration of colonisation. If transient colonisation lasts only days, proper hygiene and contact precautions may be sufficient in limiting the resident role as an MRSA vector. However, if colonisation lasts months, more aggressive measures such as decontamination may be warranted.

\section{References}

1. Gorwitz RJ, Kruszon-Moran D, McAllister SK, et al. Changes in the prevalence of nasal colonisation with Staphylococcus aureus in the United States, 2001-2004. I Infect Dis 2008; 197(9): 1226-1234.

http.dx.doi.org/10.1086/533494

2. Albrich WC, Harbarth S. Health-care workers: source, vector, or victim of MRSA? Lancet Infect Dis 2008; 8(5): 289-301. http.dx.doi.org/10.1016/S1473-3099(08)70097-5

3. Lu PL, Chin LC, Peng CF, et al. Risk factors and molecular analysis of community methicillin-resistant Staphylococcus aureus carriage. J Clin Microbiol 2005; 43(1): 132-139.

http.dx.doi.org/10.1128/JCM.43.1.132-139.2005

4. Eveillard M, Martin Y, Hidri N, et al. Carriage of methicillinresistant Staphylococcus aureus among hospital employees: prevalence, duration, and transmission to households. Infect Control Hosp Epidemiol 2004; 25(2): 114-120. http.dx.doi.org/10.1086/502360

5. Guclu E, Yavuz T, Tokmak A, et al. Nasal carriage of pathogenic bacteria in medical students: effects of clinic exposure on prevalence and antibiotic susceptibility. Eur Arch Otorhinolaryngol 2007; 264(1): 85-88. http.dx.doi.org/10.1007/s00405-006-0160-5

6. Frazee BW, Lynn J, Charlebois ED, et al. High prevalence of methicillin-resistant Staphylococcus aureus in emergency department skin and soft tissue infections. Ann Emerg Med 2005; 45(3): 311-320.

http.dx.doi.org/10.1016/j.annemergmed.2004.10.011 